\title{
Amygdala and hippocampal activity during acquisition and extinction of human fear conditioning
}

\author{
DAVID C. KNIGHT \\ University of Wisconsin, Milwaukee, Wisconsin \\ and National Institute of Mental Health, Bethesda, Maryland \\ CHRISTINE N. SMITH \\ University of Wisconsin, Milwaukee, Wisconsin \\ and University of California, San Diego, La Jolla, California \\ DOMINIC T. CHENG \\ University of Wisconsin, Milwaukee, Wisconsin \\ ELLIOT A. STEIN \\ Medical College of Wisconsin, Milwaukee, Wisconsin \\ and National Institute on Drug Abuse/Intramural Research Program, Baltimore, Maryland
}

and

FRED J. HELMSTETTER

University of Wisconsin, Milwaukee, Wisconsin

and Medical College of Wisconsin, Milwaukee, Wisconsin

\begin{abstract}
Previous functional magnetic resonance imaging (fMRI) studies have characterized brain systems involved in conditional response acquisition during Pavlovian fear conditioning. However, the functional neuroanatomy underlying the extinction of human conditional fear remains largely undetermined. The present study used fMRI to examine brain activity during acquisition and extinction of fear conditioning. During the acquisition phase, participants were either exposed to light (CS) presentations that signaled a brief electrical stimulation (paired group) or received light presentations that did not serve as a warning signal (control group). During the extinction phase, half of the paired group subjects continued to receive the same treatment, whereas the remainder received light alone. Control subjects also received light alone during the extinction phase. Changes in metabolic activity within the amygdala and hippocampus support the involvement of these regions in each of the procedural phases of fear conditioning. Hippocampal activity developed during acquisition of the fear response. Amygdala activity increased whenever experimental contingencies were altered, suggesting that this region is involved in processing changes in environmental relationships. The present data show learning-related amygdala and hippocampal activity during human Pavlovian fear conditioning and suggest that the amygdala is particularly important for forming new associations as relationships between stimuli change.
\end{abstract}

In Pavlovian fear conditioning, a neutral stimulus (conditional stimulus, or CS), usually a light or tone, is typically paired with an aversive stimulus (unconditional stimulus, or UCS) such as an electric shock. Animals learn that the CS predicts the UCS as the stimuli are repeatedly paired, and this learning is manifested as changes in behavioral and autonomic responses (Rescorla, 1988). Presentation of the CS alone provokes the expression of a

This work was supported by NIMH Fellowship MH11722 to D.C.K., NIDA Grant DA09465 to E.A.S., NIMH Grant MH50864 to F.J.H., a McDonnell Foundation grant to F.J.H., and General Clinical Research Centers Grant M01 RR00058 to the Medical College of Wisconsin. Correspondence should be addressed to F. J. Helmstetter, Department of Psychology, University of Wisconsin, P. O. Box 413, Milwaukee, WI 53201 (e-mail: fjh@uwm.edu). conditional fear response once learning has occurred. The thalamus, hippocampus, and sensory cortex each contribute to conditional response (CR) acquisition, and the amygdala is a principal site of CS-UCS convergence and critical synaptic plasticity (Bailey, Kim, Sun, Thompson, \& Helmstetter, 1999; Davis, 2000; LeDoux, 1995; Maren, 2001). In extinction, CR magnitude or probability tends to decrease as the CS is repeatedly presented without the UCS. Extinction of conditional fear probably represents the learning of a new CS-no-UCS relationship rather than the weakening of the initial CS-shock association and can be prevented by blockade of NMDA receptors within the amygdala (Baker \& Azorlosa, 1996; Falls, Miserendino, \& Davis, 1992; Meyers \& Davis, 2002; Santini, Muller, \& Quirk, 2001). Thus, the amygdala also appears to play a 
critical role in the extinction, as well as the acquisition, of conditional fear. Recent studies indicate that CR extinction relies upon both short-term (within session) and longterm (across session) memory processes (Santini et al., 2001). Short-term extinction processes appear to be mediated through amygdala-dependent circuitry, and modulation of this circuitry by the ventromedial prefrontal cortex may be critical for long-term extinction (Milad \& Quirk, 2002; Quirk, Russo, Barron, \& Lebron, 2000; Santini et al., 2001).

Imaging studies examining neural activity during acquisition of conditional fear in humans have reported learning-related changes within the amygdala and hippocampus, as well as in other brain regions (Büchel, Dolan, Armony, \& Friston, 1999; Büchel, Morris, Dolan, \& Friston, 1998; Cheng, Knight, Smith, Stein, \& Helmstetter, 2003; Knight, Cheng, Smith, Stein, \& Helmstetter, 2004; Knight, Smith, Stein, \& Helmstetter, 1999; LaBar, Gatenby, Gore, LeDoux, \& Phelps, 1998). Differential responding to CS presentations rapidly decreased in the amygdala as training continued in some of these studies (Büchel et al., 1999; Büchel et al., 1998; LaBar et al., 1998). One interpretation of these data is that the amygdala is important for initial acquisition of fear but contributes less once the response has been learned. However, procedural factors may also explain these data. Some of these studies included CS-alone trials prior to the imaging session, requiring subjects to learn a new contingency when training with the UCS began (Büchel et al., 1999; LaBar et al., 1998). Therefore, the amygdala activation observed may be associated with factors related to the altered relationships among stimuli. Our prior work on fear conditioning and fMRI has not used preconditioning exposure to the $\mathrm{CS}$, and although we have shown significant activation within a number of brain regions such as the anterior cingulate, medial thalamus, and primary visual cortices, we typically do not observe much CS-related amygdala activity during acquisition (see, e.g., Cheng et al., 2003; Knight et al., 2004; Knight et al., 1999). One possible explanation for these divergent amygdala results is the change in stimulus relationships associated with amygdala activation. In fact, the amygdala has been implicated in novelty detection (Fischer, et al., 2003; Montag-Sallaz, Welzl, Kuhl, Montag, \& Schachner, 1999) and is an important component of the attentional circuitry involved in the formation of new associations during unexpected learning experiences (Holland \& Gallagher, 1999; Holland, Han, \& Gallagher, 2000). Therefore, activation of this attentional circuitry would be expected following a change in stimulus relationships given that such changes produce a novel experience through which new associations must be formed.

Several theories have been offered to explain the involvement of the hippocampus during Pavlovian conditioning procedures. Although the hippocampus is not normally included in the neural circuitry thought to be critical for single-cue delay learning, multiple technical approaches indicate that this region is differentially active during such conditioning tasks. It is generally held that more complex conditioning procedures, including trace conditioning, discrimination reversal, and context conditioning, are dependent upon the hippocampus, whereas single-cue delay conditioning is not. Learning in these more complex procedural variants is generally disrupted by hippocampal lesions, whereas simple delay conditioning remains intact (Berger \& Orr, 1983; Moyer, Deyo, \& Disterhoft, 1990; Solomon, Vander Schaaf, Thompson, \& Weisz, 1986). However, hippocampal unit recordings show learning-related changes in neuronal activation during both simple and complex conditioning tasks (Berger \& Orr, 1983; Weiss, Kronforst-Collins, \& Disterhoft, 1996). These studies suggest that although the hippocampus is only necessary for performance in more complex conditioning procedures, it may be metabolically active during simple conditioning tasks as well. This neuronal activation may be measurable through BOLD (blood oxygen level dependent) fMRI signal changes. In an attempt to better define the role of the amygdala and hippocampus, we used fMRI during the acquisition and extinction of Pavlovian fear conditioning in healthy human subjects.

\section{METHOD}

\section{Participants}

Thirty healthy, right-handed subjects (17 women and 13 men), ranging in age from 18 to 39 (mean $\pm S E M=24.5 \pm 5.17$ years), were recruited for this study. All participants provided informed consent, and all procedures were approved by the Institutional Review Boards of the University of Wisconsin-Milwaukee and the Medical College of Wisconsin.

\section{Materials}

Functional MRI. Imaging was performed on a 1.5-Tesla GE Signa scanner using a gradient-echo, echo-planar pulse sequence. Contiguous 8-mm sagittal slices were collected (TR $=3,000 \mathrm{msec}$, $\mathrm{TE}=40 \mathrm{msec}, \mathrm{FOV}=24 \mathrm{~cm}, 64 \times 64$ matrix, $3.75 \mathrm{~mm} \times 3.75 \mathrm{~mm}$ in-plane resolution, flip angle $=90^{\circ}$ ) in a series of 86 sequential images during each 258 -sec block of stimulus presentations. Highresolution spoiled gradient recalled acquisition at steady state (SPGR) anatomical images were acquired prior to echo-planar imag-ing to allow subsequent anatomical localization of functional activation. Foam padding was used to limit head movements within a customized three-axis local gradient coil (Wong, Tan \& Hyde, 1993).

Electrical stimulus. A customized AC $(60-\mathrm{Hz})$ source was used to provide transcutaneous electrical stimulation (the UCS; 0.5 -sec duration) through two aluminum surface electrodes (2-cm diameter) placed over the right tibial nerve above the medial malleolus. The intensity of electrical stimulation was individually set for each participant. Participants determined the intensity of the electrical stimulation by rating practice trials from 0 to $5(0=$ no sensation, $5=$ painful but tolerable). The intensity of the first practice trial was equal to $0 \mathrm{~mA}$ and was gradually increased until the participant rated the stimulation as a 5 , or the stimulus intensity reached the maximal current of $7.5 \mathrm{~mA}$. Once set, the electrical stimulation was maintained at a constant intensity throughout the experiment.

Visual stimulus. A red $25-\mathrm{W}$ light bulb was flashed $(0.5 \mathrm{~Hz})$ for $15 \mathrm{sec}$ as the CS. The light bulb was housed in a black plastic light fixture, positioned $10^{\circ}$ to the right side of a fixation point (a small red light-emitting diode [LED]), mounted $5.5 \mathrm{~m}$ from the scanner. The CS was viewed with a pair of prism glasses. 
Skin conductance response (SCR). A J\&J thermal monitoring system (Model T-68) with GSR Preamp (Model IG-3) was used to monitor each participant's skin conductance throughout the experiment. Skin conductance was monitored with a pair of surface gel cup electrodes $(\mathrm{Ag} / \mathrm{AgCl}, 1-\mathrm{cm}$ diameter) attached $2 \mathrm{~cm}$ apart on the sole of the participant's left foot. Although SCR is traditionally monitored from the palm of the hand, compatibility issues between the $\mathrm{SCR}$ and MRI equipment required measurement from this alternative location. All skin conductance data were range corrected by dividing each participant's raw skin conductance by his or her mean skin conductance level across each training block. SCR was calculated as the peak response that occurred during the 15-sec CS presentation and was expressed as a percentage of the evoked SCR to the first conditioning trial. SCR data were averaged for each training block of the acquisition and extinction phases of the study.

\section{Procedure}

Participants were randomly assigned to one of two groups (paired, $n=20$; control, $n=10$ ). During the acquisition phase, paired subjects received CS $(15 \mathrm{sec})$ and $\mathrm{UCS}(0.5 \mathrm{sec}$; intertrial interval [ITI] $=$ $30 \mathrm{sec})$ presentations that coterminated, whereas the control group received explicitly unpaired CS $(15 \mathrm{sec})$ and UCS $(0.5 \mathrm{sec}$; ITI = $30 \mathrm{sec}$, ISI $=15 \mathrm{sec}$ ) presentations. Following four blocks of acquisition trials ( 5 trials per block), half of the paired group subjects were randomly assigned to a new extinction group. During the extinction phase, the paired group $(n=10)$ continued to receive the same CS-UCS pairings, but the extinction $(n=10)$ and control $(n=10)$ groups were presented light alone (see Figure 1). Subjective pain ratings of the UCS were obtained after each block of trials.

\section{Functional MRI Analysis}

Functional image analyses were performed using AFNI Version 2.2 (Cox \& Hyde, 1997). In-plane motion correction and edgedetection algorithms were applied to the functional data. Activations within the amygdala and hippocampus were identified through cross-correlation analysis with a reference waveform representing the temporal pattern of visual stimulation. The reference waveform was shifted by one image to account for the delay in the hemodynamic response. Given the anticipated hemodynamic delay and rise time, BOLD signal changes elicited by UCS presentation were excluded from this analysis. Functional and anatomical images for each subject were transformed into stereotactic coordinate space relative to the line between the anterior and posterior commisures (Talairach \& Tournoux, 1988). Functional maps were spatially blurred using a 3.0-mm RMS Gaussian filter. A two-factor analysis of variance (ANOVA) was performed on a voxel-wise basis, resulting in an $F$ statistic $(F>3.07)$ that was used to restrict additional analyses to those voxels that were significantly activated $(p<.01)$. Changes in the pattern of activation across the conditioning session were evaluated by $t$ test comparisons $(t>2.89, p<.01)$.

\section{Acquisition}
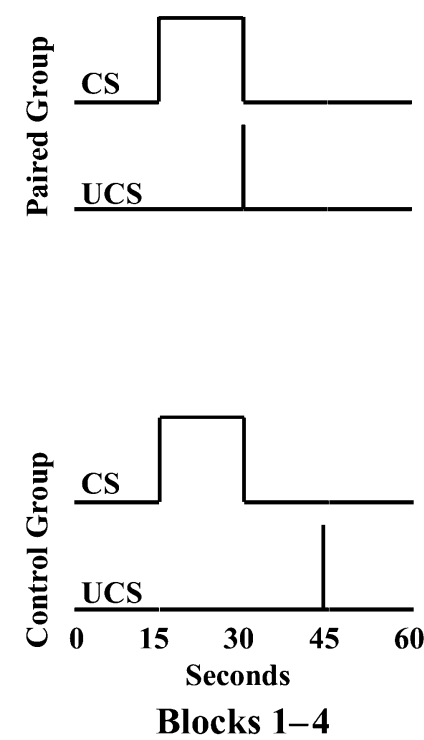

Extinction

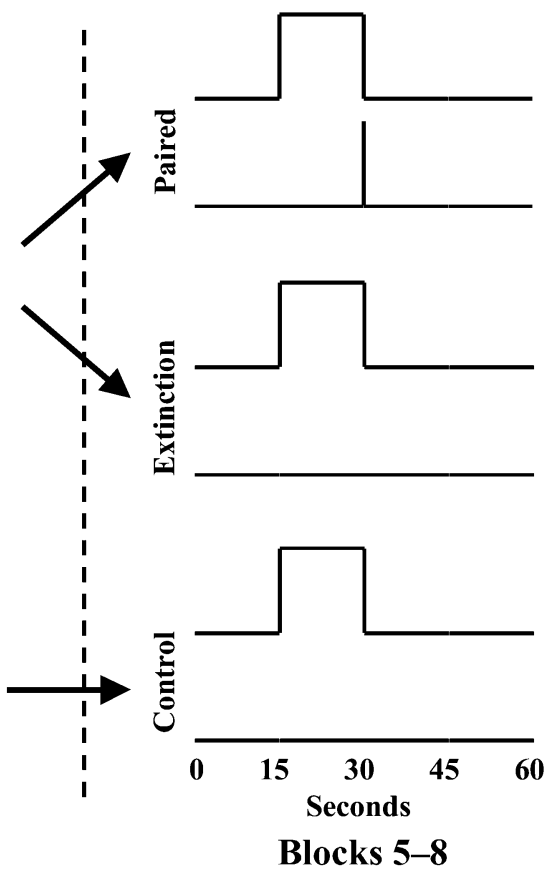

Figure 1. Illustration of the experimental design. Participants were randomly assigned to one of two groups (paired group, $n=20$, or control group, $n=10$ ) that received four blocks of stimulus presentations ( 5 trials per block) during the acquisition phase. Acquisition phase: Paired subjects received coterminating CS and UCS presentations, whereas control subjects received explicitly unpaired CS and UCS presentations. During the extinction phase, the paired group was divided, and half of the subjects (paired group, $n=10$ ) continued to receive the same CS-UCS pairings, whereas the other half (extinction group, $n=10)$ and the control group $(n=10)$ were presented with the light alone. 


\section{RESULTS}

\section{Electrical Stimulation}

All groups received UCS presentations of similar intensity (mean $\pm S E M=$ paired, $3.78 \pm 0.76 \mathrm{~mA}$; extinction, $4.21 \pm 0.63 \mathrm{~mA}$; control, $3.72 \pm 0.6 \mathrm{~mA} ; F<1$ ).

\section{Pain Rating}

All groups reported similar pain ratings during the acquisition phase of the experiment (mean $\pm S E M=$ paired, $3.95 \pm 0.11$; control, $3.68 \pm 0.18 ; F<1$ ). Paired subjects provided comparable pain ratings during both the acquisition and extinction phases, whereas extinction and control subjects reported essentially no pain during the extinction phase when no UCS was presented (mean \pm $S E M=$ paired, $3.82 \pm 0.18$; extinction, $0.07 \pm 0.07$; control, $0.12 \pm 0.07 ; F=84.46, p<.05)$.

\section{Skin Conductance Response}

Comparisons of SCR (see Figure 2) during the acquisition phase demonstrated that CRs were greater for subjects who received paired CS-UCS presentations as opposed to controls $(t=2.19, p<.05)$. These differences confirm that the CS provoked a conditional fear response in paired but not in control subjects. During the extinction phase, subjects within the extinction and control groups received presentations of the light alone, whereas the paired group continued to receive the same CS-UCS pairings. Large conditioned SCRs were maintained in paired group subjects, as opposed to controls $(t=2.30, p<.05)$, during this phase of the study, whereas CR amplitude for extinction group subjects fell to an intermediate level that did not differ from that of the paired $(t=1.10)$ or control $(t=1.15)$ groups. CR timing (mean $\pm S E M=7.68 \pm$ $0.57 \mathrm{sec}$ ) was consistent with second interval responding, which is often used as an index of learning CS-UCS associations (Prokasy \& Raskin, 1973; Wolter \& Lachnit, 1993).

\section{Functional MRI Results}

An ANOVA revealed the development of significant stimulus-evoked activity within the bilateral amygdala (Talairach coordinate system: right, 19, $-11,-19$; left, $-22,-6,-19)$ and left hippocampus (Talairach coordinate system: $-29,-15,-12$ ) over the course of training. Amygdala activation was similar for all groups during the acquisition phase of the study (Figure 3A). However, as can be seen in Figure 3B, right amygdala activity increased in subjects receiving CS alone trials (extinction and control groups) during the extinction phase of the experiment, whereas left amygdala activity decreased in these groups. Paired subjects showed no change in amygdala activation. As is illustrated in Figure 3C, differences in right amygdala activation quickly returned to pre-extinction phase levels, whereas differences in left amygdala activation gradually decreased across the extinction phase.

Differences in left hippocampal activation developed over the course of acquisition and were maintained into the extinction phase of the experiment. As can be seen in Figure 4A, hippocampal activation was similar for all groups during the first training block. However, subjects receiving paired CS-UCS presentations showed greater left hippocampal activity than did controls by the last block of acquisition (see Figure 4A). Figure 4B demonstrates that left hippocampal activity decreased in extinction subjects as they began the extinction phase of the study, but that no change in hippocampal activity was revealed for
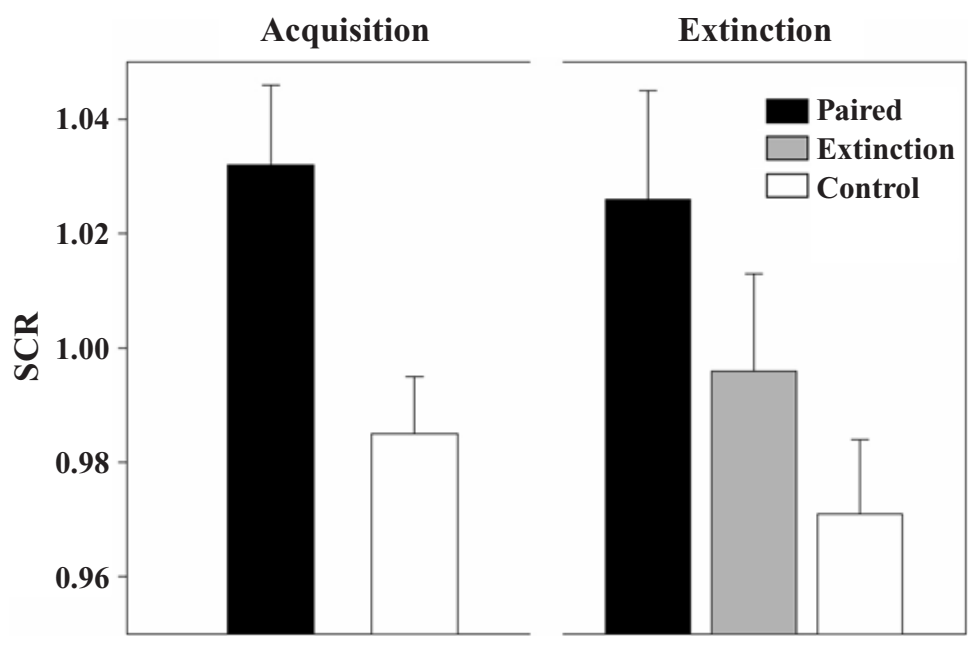

Figure 2. SCR for paired, extinction, and control groups during the acquisition and extinction phases. During the acquisition phase, the paired group $(n=$ 20) produced larger CRs than did controls $(n=10)$. During the extinction phase, the paired group $(n=10)$ continued to produce large CRs, whereas the extinction group's $(n=10)$ CRs fell to an intermediate level in comparison with the small responses elicited by controls $(n=10)$. 


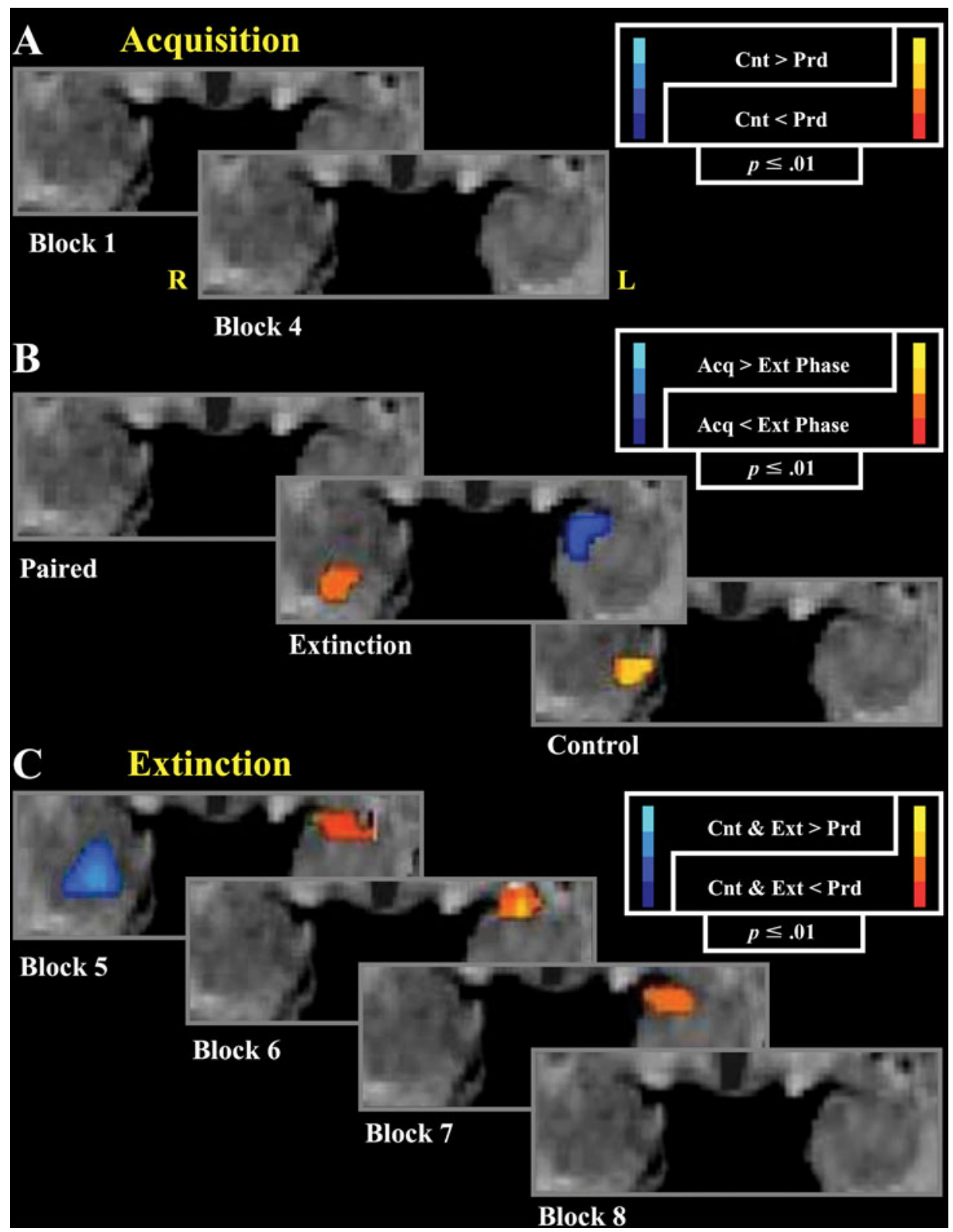

Figure 3. Amygdala activity. (A) Paired (Prd) compared with control (Cnt) group activation on the first and last blocks of the acquisition phase. No differences in amygdala activity were demonstrated between groups during this phase of the study. (B) Comparison of amygdala activity during the last stimulus block of the acquisition phase and first block of the extinction phase for each group. Right amygdala activity increased in extinction (Ext) and Cnt subjects, and left amygdala activity decreased for the Ext group during the extinction phase of the study. Subjects who continued to receive the same CS-UCS pairings (Prd group) throughout the study showed no change in amygdala activation. (C) Comparison of group differences (Cnt \& Ext vs. Prd) during the extinction phase of the study. A brief increase in right amygdala activity coincided with the initiation of extinction trials for Cnt and Ext subjects and then quickly returned to pre-extinction phase levels. In contrast, left amygdala activity, during extinction, was greater for the paired group than for subjects receiving the CS alone (Cnt \& Ext), but gradually diminished across the extinction phase.

the paired or the control groups. Figure 4C shows differences in hippocampal activity for paired versus extinction and control groups for each block of the extinction phase of the experiment. Left hippocampal activity was greater in paired subjects initially, but it decreased over the course of the extinction phase. 


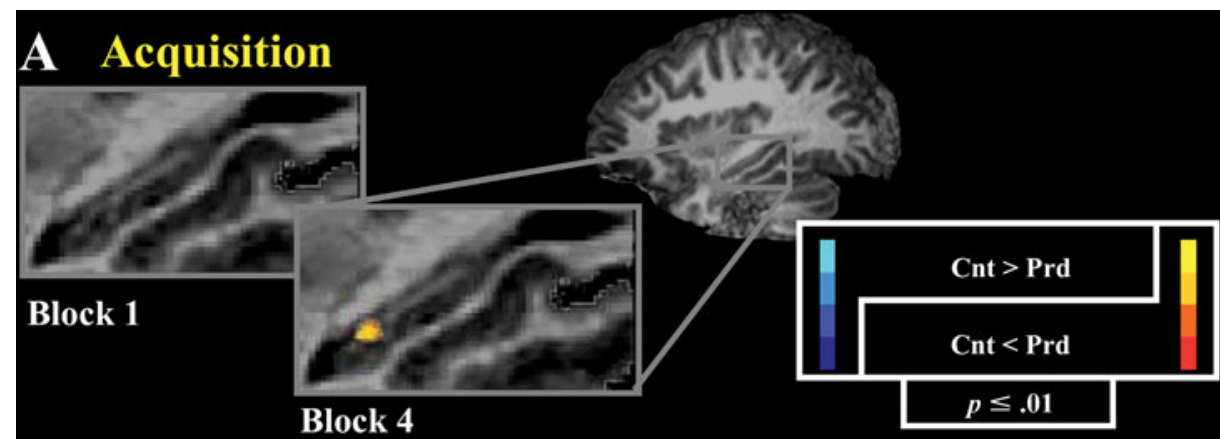

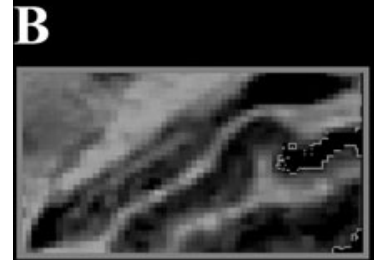

Paired

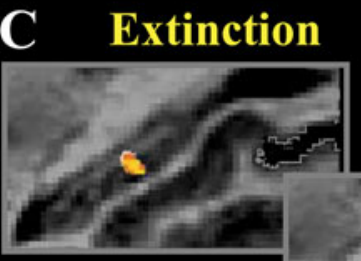

Block 5
Extinction

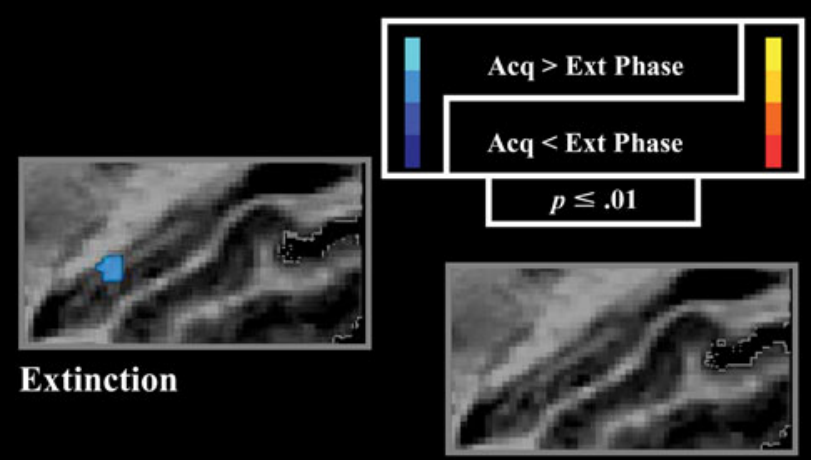

\section{Control}

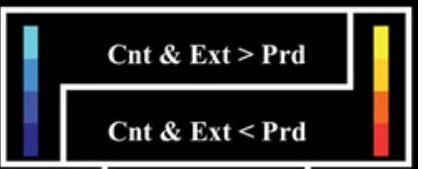

$p \leq .01$

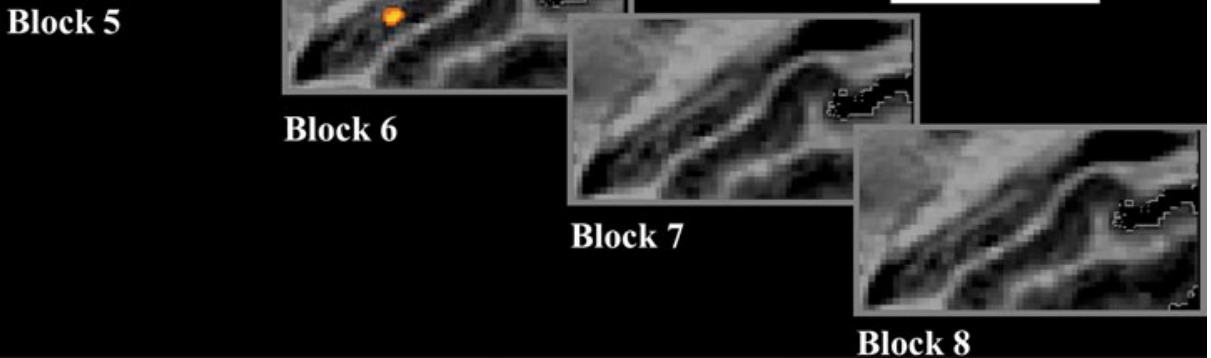

Figure 4. Left hippocampal activity. (A) Paired (Prd) compared with control (Cnt) group activation on the first and last blocks of the acquisition phase. Although left hippocampal activity was similar for both groups at the beginning of the session, significant differences (Prd $>$ Cnt) developed with repeated training. (B) Comparison of left hippocampal activity during the last acquisition block and first extinction block for each group. Activity decreased during the extinction phase for the extinction (Ext) group, whereas no change was observed for Cnt and Prd subjects. (C) Comparison of group differences (Cnt \& Ext vs. Prd) during the extinction phase of the study. Left hippocampal activity remained greater for Prd subjects initially, but habituated across the extinction phase of the study.

\section{DISCUSSION}

\section{Amygdala}

The amygdala is a critical component of the neural circuit thought to mediate acquisition of conditional fear. Afferent projections carrying information about the CS and UCS converge within subnuclei of the amygdala, and pro- jections from the amygdala to brainstem targets appear to be important for the expression of critical autonomic and behavioral responses (Davis, 2000; Helmstetter, Tershner, Poore, \& Bellgowan, 1998; LeDoux, 1995; Price \& Amaral, 1981). Although most of the research exploring the amygdala's contribution to conditional fear has been conducted with laboratory animals, functional brain imaging 
studies support the amygdala's importance for this type of learning in humans as well (Büchel et al., 1999; Büchel et al., 1998; Cheng et al., 2003; LaBar et al., 1998). In the present study, differential task-related amygdala activation was only observed following a change in stimulus relationships, which is consistent with the view that this region is an important component of the attentional circuitry supporting the formation of new associations when relationships between stimuli change (Holland \& Gallagher, 1999; Holland et al., 2000). These data provide further evidence that the amygdala is critically involved in human Pavlovian fear conditioning and suggest that such differential amygdala activity may be related to specific features of the training procedure that require the learning of new relationships between stimuli.

Previous fMRI fear conditioning research has typically observed unilateral activation of the right amygdala (Büchel et al., 1999; Büchel et al., 1998; Cheng et al., 2003; LaBar et al., 1998). The right amygdala activation observed in the present study is largely consistent with this prior work. LaBar et al. observed amygdala activity during both early acquisition and extinction. In the present study, however, differential amygdala activity was observed only during extinction. One possible explanation for these differences is the use of CS habituation trials prior to training in the previous study. The differential activation observed during early acquisition trials may have been driven by the change in contingency for the $\mathrm{CS}+$, but not $\mathrm{CS}-$, during the transition from habituation to acquisition rather than by acquisition of the CR itself. In the present study, subjects had no prior exposure to target stimuli. Therefore, all CS presentations were initially novel and appear to have produced similar levels of right amygdala activation. Other fMRI fear learning research has observed that the peak amygdala response follows stimulus onset and then attenuates after several seconds of long-duration (e.g., $18 \mathrm{sec}$ ) stimuli (Phelps et al., 2001). The CS duration in the present study approximated the length of time during which amygdala responses were maintained in prior fMRI work. However, our analysis may be insensitive to more rapid within-trial attenuation of amygdala activity. Future studies using long-duration CS periods should explore dynamic within-trials changes of the amygdala response.

Research with laboratory animals indicates that the amygdala is also important for extinction of conditional fear (Baker \& Azorlosa, 1996; Falls et al., 1992). Our data lend support to this concept. During the first block of the extinction phase, right amygdala activity increased when extinction group subjects were presented the light alone. In contrast, paired group subjects who continued to receive the same CS-UCS pairings throughout both phases showed no change in amygdala activation. Further, our data suggest that this amygdala activity may have been related to changes in stimulus relationships, as is seen when subjects are switched from acquisition to extinction trials. Control subjects who received unpaired training and then had UCS presentations withheld in the final phase of the experiment also showed an increase in right amygdala activity that was similar to the response observed in extinction subjects. By design, control group subjects should have no CR to extinguish since the treatment they received does not support excitatory conditioning. Therefore, the transient amygdala activation observed in control subjects cannot be generated by a process exclusively related to extinction of the CR. An alternative interpretation is that the right amygdala activity for both the control and extinction groups was driven by the change in stimulus relationships that occurred during the transition from the acquisition phase to the extinction phase. This interpretation is consistent with suggestions that the amygdala is involved in orienting and attentional processes during Pavlovian conditioning and may be involved in the establishment of new associations following the violation of current expectancies (Fischer et al., 2003; Holland \& Gallagher, 1999; Holland et al., 2000; Montag-Sallaz et al., 1999). Each of these processes is likely to be present during a conditioning session and may be important for forming CS-UCS associations. However, it will be important for future studies to determine which of these processes drive amygdala activity, so that we may gain a better understanding of this region's relative contributions to the various processes involved in Pavlovian fear conditioning.

A distinct pattern of left amygdala activity was also observed in the present study. Beginning with onset of the extinction phase, subjects who continued to receive paired CS-UCS presentations showed greater left amygdala activation than did subjects who received the CS alone (i.e., the extinction and control groups). This activation of the left amygdala is consistent with previous fear learning studies that have ascribed higher level cognitive processes to this region. Activation of the left amygdala has been associated with the cognitive representation of fear acquired through verbal instructions (Phelps et al., 2001), and other work has suggested that left amygdala activation is associated with awareness of the CS-UCS relationship (Morris, Öhman, \& Dolan, 1998). Interestingly, the pattern of left amygdala activity obtained in those studies appeared to mirror the pattern of activation within the left hippocampus in the present study. Although left amygdala activity was delayed (by one block) relative to the activation pattern observed in the left hippocampus, this similarity in activation patterns may reflect the interaction of the amygdala and hippocampus during the encoding of fear memories (Richardson, Strange, \& Dolan, 2004).

\section{Hippocampus}

Differential hippocampal activity was also observed in the present study. Paired group subjects showed greater left hippocampal activation than did their controls by the end of the acquisition phase. These differences were maintained through the first two blocks of the extinction phase. In contrast, the participants who switched from paired CS-UCS presentations to CS-alone trials (i.e., the extinction group) showed a significant decrease in hippocampal activity during early trials of the extinction phase. Although the hippocampus is not generally considered crit- 
ical for single-cue delay conditioning, the present results support previous observations of hippocampal activity during delay eyeblink and fear conditioning (Berger, Rinaldi, Weisz, \& Thompson, 1983; Blaxton, Zeiffiro, \& Gabrieli, 1996; Knight et al., 2004; Ramnani, Toni, Josephs, Ashburner, \& Passingham, 2000; Weiss et al., 1996). Furthermore, decreased hippocampal responsiveness, similar to that seen in the extinction group in the present study, has been previously demonstrated with behavioral extinction in animal models (Berger \& Thompson, 1982; Segal, 1973).

The hippocampus has prominent reciprocal connections with the amygdala as well as cingulate, prefrontal, and temporal cortical areas known to be important for certain forms of memory (Amaral \& Cowan, 1980; Amaral \& Insausti, 1992). The hippocampus and medial temporal cortex have been specifically implicated in explicit or declarative memory processes (awareness or conscious recollection of facts and events), which are often contrasted with implicit or nondeclarative memory (a skill, habit, or other behavior that is unconsciously performed; see, e.g., Clark, Manns, \& Squire, 2002; Milner, Squire, \& Kandel, 1998; Squire, 1992). Left lateralization of hippocampal activity, similar to that demonstrated in the present study, has been previously observed in fMRI conditioning research (Knight et al., 2004; Ramnani et al., 2000). Left hippocampal activation has been associated with declarative memory processes that involve matching a stimulus to a mnemonic representation (Iidaka et al., 2003). Furthermore, concurrent activation of the left hippocampus and amygdala may reflect associative processing of emotional material in declarative memory (Killgore, Casasanto, Yurgelun-Todd, Maldjian, \& Detre, 2000). Although successful performance in single-cue Pavlovian conditioning does not require conscious awareness of programmed contingencies (e.g., Bechara et al., 1995; Clark \& Squire, 1998), declarative memory processes are clearly engaged in normal subjects during standard conditioning tasks (Knight et al., 2004; Knight, Nguyen, \& Bandettini, 2003). Therefore, it is possible that the hippocampal activity seen in the present study may reflect the development of declarative knowledge of the CS-UCS relationship.

In sum, the present study explored amygdala and hippocampal activation during the acquisition and extinction of human Pavlovian fear conditioning. These results highlight the amygdala's role in the short-term extinction of conditional fear and suggest that region is particularly sensitive to changes in environmental contingencies requiring new learning. Our study focused explicitly on short-term extinction processes that occur within a single session. Recent studies suggest that long-term retention $(>24 \mathrm{~h})$ of extinction memory requires processes that occur within the prefrontal cortex between training sessions (Milad \& Quirk, 2002; Quirk et al., 2000; Santini et al., 2001). Additional imaging work is currently exploring the neural mechanisms that support long-term extinction of conditional fear (e.g., Richards, Cheng, Thomas, Smith, \& Helmstetter, 2003).

\section{REFERENCES}

Amaral, D. G., \& Cowan, W. M. (1980). Subcortical afferents to the hippocampal formation in the monkey. Journal of Computational Neurology, 189, 573-591.

Amaral, D. G., \& Insausti, R. (1992). Retrograde transport of D-[ $\left.{ }^{3} \mathrm{H}\right]-$ aspartate injected into the monkey amygdaloid complex. Experimental Brain Research, 88, 375-388.

Bailey, D. J., Kim, J. J., Sun, W., Thompson, R. F., \& Helmstetter, F. J. (1999). Acquisition of fear conditioning in rats requires the synthesis of mRNA in the amygdala. Behavioral Neuroscience, 113, 276282.

BAKer, J. D., \& Azorlosa, J. L. (1996). The NMDA antagonist MK801 blocks the extinction of Pavlovian fear conditioning. Behavioral Neuroscience, 110, 618-620.

Bechara, A., Tranel, D., Damasio, H., Adolphs, R., Rockland, C., \& Damasio, A. (1995). Double dissociation of conditioning and declarative knowledge relative to the amygdala and hippocampus in humans. Science, 269, 1115-1118.

BERGER, T. W., \& ORR, W. B. (1983). Hippocampectomy selectively disrupts discrimination reversal conditioning of the rabbit nictitating membrane response. Behavioural Brain Research, 8, 49-68.

Berger, T. W., Rinaldi, P. C., Weisz, D. J., \& THOMPSON, R. F. (1983). Single-unit analysis of different hippocampal cell types during classical conditioning of rabbit nictitating membrane response. Journal of Neurophysiology, 50, 1197-1219.

BERGER, T. W., \& THOMPSON, R. F. (1982). Hippocampal cellular plasticity during extinction of classically conditioned nictitating membrane behavior. Behavioural Brain Research, 4, 63-67.

Blaxton, T. A., Zeiffiro, T. A., \& Gabrieli, J. D. E. (1996). Functional mapping of human learning: A positron emission tomography activation study of eyeblink conditioning. Journal of Neuroscience, 16, 4032-4040.

BüCHel, C., Dolan, R. J., Armony, J. L., \& Friston, K. J. (1999). Amygdala-hippocampal involvement in human aversive trace conditioning revealed through event-related functional magnetic resonance imaging. Journal of Neuroscience, 19, 10869-10876.

Büchel, C., Morris, J., Dolan, R. J., \& Friston, K. J. (1998). Brain systems mediating aversive conditioning: An event-related fMRI study. Neuron, 20, 947-957.

Cheng, D. T., KNight, D. C., Smith, C. N., Stein, E. A., \& HelmstetTER, F. J. (2003). Functional MRI of human amygdala activity during Pavlovian fear conditioning: Stimulus processing versus response expression. Behavioral Neuroscience, 117, 3-10.

Clark, R. E., Manns, J. R., \& SQuire, L. R. (2002). Classical conditioning, awareness, and brain systems. Trends in Cognitive Sciences, 6, 524-531.

CLARK, R. E., \& SQUIRE, L. R. (1998). Classical conditioning and brain systems: The role of awareness. Science, 280, 77-81.

Cox, R. W., \& HydE, J. S. (1997). Software tools for the analysis and visualization of f MRI data. NMR in Biomedicine, 10, 171-178.

DAVIS, M. (2000). The role of the amygdala in conditioned and unconditioned fear and anxiety. In J. P. Aggleton (Ed.), The amygdala: $A$ functional analysis (2nd ed., pp. 213-287). New York: Oxford University Press.

Falls, W. A., Miserendino, M. J., \& Davis, M. (1992). Extinction of fear-potentiated startle: Blockade by infusion of an NMDA antagonist into the amygdala. Journal of Neuroscience, 12, 854-863.

Fischer, H., Wright, C. I., Whalen, P. J., McInerney, S. C., Shin, L. M., \& RAUCH, S. L. (2003). Brain habituation during repeated exposure to fearful and neutral faces: A functional MRI study. Brain Research Bulletin, 59, 387-392.

Helmstetter, F. J., Tershner, S. A., Poore, L. H., \& Bellgowan, P. S. (1998). Antinociception following opioid stimulation of the basolateral amygdala is expressed through the periaqueductal gray and rostral ventromedial medulla. Brain Research, 779, 104-118.

Holland, P. C., \& Gallagher, M. (1999). Amygdala circuitry in attentional and representational processes. Trends in Cognitive Sciences, 3, 65-73.

Holland, P. C., Han, J., \& Gallagher, M. (2000). Lesions of the 
amygdala central nucleus alter performance on a selective attention task. Journal of Neuroscience, 20, 6701-6706.

IidAKa, T., TERASHima, S., Yamashita, K., OKADA, T., Sadato, N., \& YonekuRA, Y. (2003). Dissociable neural responses in the hippocampus to the retrieval of facial identity and emotion: An eventrelated fMRI study. Hippocampus, 13, 429-436.

Killgore, W. D., Casasanto, D. J., Yurgelun-Todd, D. A., MaldJiAN, J. A., \& Detre, J. A. (2000). Functional activation of the left amygdala and hippocampus during associative encoding. NeuroReport, 11, 2259-2263.

Knight, D. C., Cheng, D. T., Smith, C. N., Stein, E. A., \& HelmSTETTER, F. J. (2004). Neural substrates mediating human delay and trace fear conditioning. Journal of Neuroscience, 24, 218-228.

Knight, D. C., Nguyen, H. T., \& BandetTini, P. A. (2003). Expression of conditional fear with and without awareness. Proceedings of the National Academy of Sciences, 100, 15280-15283.

KNight, D. C., Smith, C. N., Stein, E. A., \& Helmstetter, F. J. (1999). Functional MRI of human Pavlovian fear conditioning: Patterns of activation as a function of learning. NeuroReport, 10, 3665-3670.

LaBar, K. S., Gatenby, J. C., Gore, J. C., LeDoux, J. E., \& Phelps, E. A. (1998). Human amygdala activation during conditioned fear acquisition and extinction: A mixed-trial study. Neuron, 20, 937-945.

LeDoux, J. (1995). Emotion: Clues from the brain. Annual Review of Psychology, 46, 209-235.

MAREN, S. (2001). Neurobiology of Pavlovian fear conditioning. Annual Review of Neuroscience, 24, 897-931.

MEYERS, K. M., \& DAVIS, M. (2002). Behavioral and neural analysis of extinction. Neuron, 26, 567-584.

MiLAD, M. R., \& QUIRK, J. (2002). Neurons in the medial prefrontal cortex signal memory for fear extinction. Nature, 420, 70-74.

Milner, B., SQuire, L. R., \& Kandel, E. R. (1998). Cognitive neuroscience and the study of memory. Neuron, 20, 445-468.

Montag-Sallaz, M., Welzl, H., Kuhl, D., Montag, D., \& SchachNER, M. (1999). Novelty-induced increased expression of immediateearly genes c-fos and arg 3.1 in the mouse brain. Journal of Neurobiology, 38, 234-246.

MORRIS, J. S., ÖHMAN, A., \& Dolan, R. J. (1998). Conscious and unconscious emotional learning in the human amygdala. Nature, $\mathbf{3 9 3}$ 467-470.

Moyer, J. R., Deyo, R. A., \& Disterhoft, J. F. (1990). Hippocampectomy disrupts trace eye-blink conditioning in rabbits. Behavioral Neuroscience, 104, 243-252.

Phelps, E. A., O'Connor, K. J., Gatenby, J. C., Gore, J. C., GrilLION, C., \& DAvis, M. (2001). Activation of the left amygdala to a cognitive representation of fear. Nature Neuroscience, 4, 437-441.

Price, J. L., \& Amaral, D. G. (1981). An autoradiographic study of the projections of the central nucleus of the monkey amygdala. Journal of Neuroscience, 1, 1242-1259.
ProKasy, W. F., \& RASKIN, D. C. (1973). Electrodermal activity in psychological research. New York: Academic Press.

Quirk, G. J., Russo, G. K., Barron, J. L., \& Lebron, K. (2000). The role of ventromedial prefrontal cortex in the recovery of extinguished fear. Journal of Neuroscience, 20, 6225-6231.

Ramnani, N., Toni, I., Josephs, O., Ashburner, J., \& Passingham, R. E. (2000). Learning- and expectation-related changes in the human brain during motor learning. Journal of Neurophysiology, 84, 30263035.

ResCorla, R. (1988). Behavioral studies of Pavlovian conditioning. Annual Review of Neuroscience, 11, 329-352.

Richards, J. A., Cheng, D. T., Thomas, T., Smith, C. N., \& HelmSTETTER, F. J. (2003, November). Frontal lobe activity during acquisition, extinction, and retrieval of fear conditioning. Society for Neuroscience Abstracts. Available at http://apu.sfn.org/

Richardson, M. P., Strange, B. A., \& Dolan, R. J. (2004). Encoding of emotional memories depends on amygdala and hippocampus and their interactions. Nature Neuroscience, 7, 278-285.

SAntini, E., Muller, R. U., \& Quirk, G. J. (2001). Consolidation of extinction learning involves transfer from NMDA-independent to NMDA-dependent memory. Journal of Neuroscience, 21, $9009-9017$.

SEGAL, M. (1973). Flow of conditioned responses in limbic telencephalic system of the rat. Journal of Neurophysiology, 36, 840-854.

Solomon, P. R., Vander SchaAf, E. R., Thompson, R. F., \& Weisz, D. J. (1986). Hippocampus and trace conditioning of the rabbit's classically conditioned nictitating membrane response. Behavioral Neuroscience, 100, 729-744.

SQUire, L. R. (1992). Memory and the hippocampus: A synthesis from findings with rats, monkeys, and humans. Psychological Review, 99, 195-231.

TALAIRACH, J., \& Tournoux, P. (1988). Co-planar stereotaxic atlas of the human brain: 3-dimensional proportional system. An approach to cerebral imaging. Stuttgart: Thieme.

Weiss, C., Kronforst-Collins, M. A., \& Disterhoft, J. F. (1996). Activity of hippocampal pyramidal neurons during trace eyeblink conditioning. Hippocampus, 6, 192-209.

Wolter, J., \& LACHNIT, H. (1993). Are anticipatory first and second interval skin conductance responses indicators of predicted aversiveness? Integrative Physiological \& Behavioral Science, 28, 163-166.

Wong, E. C., TAN, G., \& HyDE, J. S. (1993). A quadrature transmitreceive endcapped birdcage coil for imaging of the human head at $125 \mathrm{MHz}$. Proceedings of the International Society for Magnetic Resonance in Medicine (12th Annual Meeting), p. 1344.

(Manuscript received January 6, 2004; revision accepted for publication April 4, 2004.) 